\title{
Guest editorial: special issue on search and pursuit-evasion with mobile robots
}

\author{
Geoffrey A. Hollinger • Timothy H. Chung • \\ Volkan Isler
}

Received: 30 July 2011 / Accepted: 17 August 2011 / Published online: 1 September 2011

(C) Springer Science+Business Media, LLC 2011

Search problems (in which the objective is to find possibly mobile targets) and pursuit-evasion games (where one or more pursuers try to capture an evader who, in turn, tries to avoid capture) both have long histories in mathematics, operations research, and computer science. Classical results were sometimes driven by mere intellectual curiosity but more often by the wide range of applications, mainly in military settings. Only recently, however, have such tasks been performed by robotic systems. The increased capabilities of mobile robots are enabling autonomous search and rescue, robotic surveillance, unmanned reconnaissance, and other technologies. Beyond these applications, search and pursuit-evasion missions serve as catalysts for robotics research. That is, to develop autonomous systems for these tasks, it is necessary to build on classical tools and develop new ones which are capable of dealing with environmental complexity, sensing limitations, communication constraints, and robustness issues.

The primary aim of this special issue entitled Search and Pursuit-Evasion with Mobile Robots is to bridge the gap be-

\section{G.A. Hollinger $(\bowtie)$}

Computer Science Department, Viterbi School of Engineering, University of Southern California, Los Angeles, CA, USA

e-mail: gahollin@usc.edu

T.H. Chung

Department of Systems Engineering,

Graduate School of Engineering and Applied Sciences,

Naval Postgraduate School, Monterey, CA, USA

e-mail: thchung@nps.edu

V. Isler

Department of Computer Science and Engineering,

College of Science and Engineering, University of Minnesota,

Minneapolis, MN, USA

e-mail: isler@cs.umn.edu tween theoretical analysis and emerging applications. We start the issue with an article which surveys results related to robotic search and pursuit-evasion. The following six articles provide both new theoretical results and experimental analyses of robotic systems performing search and pursuitevasion tasks. We now summarize the articles included in this special issue.

The first article titled "Search and Pursuit-Evasion in Mobile Robotics: A Survey" is by the guest editors Chung, Hollinger, and Isler. ${ }^{1}$ It provides a summary of work in search and pursuit-evasion related to robotics applications. The article focuses on two instances of the search problem: one-sided search, typically characterized by a probabilistically modeled non-adversarial target, and adversarial search and capture, characterized by a target that actively avoids capture and may have knowledge of the pursuer's strategy. The article reviews key classical results, examines recent experimental results on robotic systems, and discusses open problems for future research.

The next article "Algorithms and Complexity Results for Graph-Based Pursuit Evasion" by Borie, Tovey, and Koenig provides new results on the computational complexity of pursuit-evasion problems on graphs. They examine the complexity of minimizing the search distance, search time, and number of searchers when a worst-case target is pursued on a graphical representation of the environment. Their results provide theoretical limits that assist in algorithm design in these domains. The authors also provide a new efficient algorithm for pursuit-evasion when the environment representation is a tree.

In their article "A Mixed Integer Linear Programming Approach to Pursuit Evasion Problems with Optional Con-

\footnotetext{
${ }^{1}$ We thank the Editor-in-Chief Gaurav S. Sukhatme for handling the review process for the survey article.
} 
nectivity Constraints," Thunberg and Ögren present an approach for solving the adversarial search problem using a MILP formulation. Their MILP formulation allows for team constraints, such as connectivity, to be incorporated into the search schedule. In addition, the formulation of pursuitevasion as a MILP facilitates the use of existing and highlyoptimized solvers. Their method generates the search schedule using a receding horizon method, which provides scalability to large environments and team sizes.

Murrieta-Cid, Ruiz, Marroquin, Laumond, and Hutchinson explore the problem of pursuit-evasion in continuous space in their article "Tracking an Omnidirectional Evader with a Differential Drive Robot." They examine the case where the pursuer and evader have different capabilities: the evader's velocity is bounded only by speed, whereas the pursuer has both non-holonomic constraints and speed limitations. The authors provide lower bounds on the pursuer's speed to track the evader, and they derive optimal strategies for both the pursuer and the evader to track and avoid respectively. The incorporation of non-holonomic constraints into tracking strategies moves towards direct implementation of continuous pursuit-evasion strategies on robotic vehicles.

In "Spatial Approaches to Broadband Jamming in Heterogenous Mobile Networks: A Game-Theoretic Approach," Bhattacharya and Başar examine the problem in which a mobile agent jams the communication network of several unmanned vehicles. They provide a game-theoretic formulation and use Isaacs' approach to derive the necessary conditions to find saddle-point strategies for the unmanned vehicles. They provide numerical simulations for various conditions and utilize these to generate optimal trajectories. The jamming evasion problem, while relatively new to the pursuit-evasion literature, provides a novel application domain of significant interest.

Modeling target motion is an important, and often ignored aspect of search and pursuit-evasion problems. Due to the difficulty of modeling complex motion, random walks or other simple models are typically favored. Joseph, DoshiVelez, Huang, and Roy provide a new method for modeling motion patterns of targets in "A Bayesian Nonparameric Approach to Modeling Motion Patterns." They show how a novel variant of Gaussian Processes can be used to develop complex motion models without expert knowledge. Their method avoids the difficult problem of parameterizing the target's motion pattern and operates with minimal training data. They apply their technique to car-tracking in the greater Boston area, which provides immediate applicability to surveillance applications.

The final article "Exploration Strategies based on MultiCriteria Decision Making for Searching Environments in Rescue Operations" by Nicola Basilico and Francesco Amigoni provides a formal approach for optimizing multiple performance metrics in the context of robotic exploration. When searching for a target or exploring an environment, there are often competing objectives, such as minimizing energy consumption, search time, and risk to the autonomous searcher. The authors provide principled methods for combining multiple objective functions, and they validate their approach in the emerging domain of autonomous search and rescue.

Many helpful souls have assisted with this special issue. We thank the authors for submitting their high-quality work and for revising and re-revising on schedule. We gratefully acknowledge the reviewers for providing feedback and critiques that improved both the technical quality and clarity of the articles. Finally, we thank the editor-in-chief Gaurav S. Sukhatme, the journal office, and the publishers for their invaluable assistance. We sincerely hope that the resulting issue is insightful and informative for both experienced pursuit-evasion researchers and those entering the field. 